\title{
Influence Analysis on Heat Effect of Size Parameter for Mold Heat System of Large-Scale Wind Turbine Blade
}

\author{
Pengwen Sun ${ }^{*}$, Jiajia Gao, Zhejian Xing and Yuan Zhang
}

College of Mechanical Engineering, Inner Mongolia University of Technology, Hohhot, 010051, China

\begin{abstract}
Wind turbine blade is the key component of wind power system. Curing is one of the important processes in its production of large-scale composite wind turbine blade. Mold heating system of circulating water affects the quality of resin curing and the productive efficiency of turbine blade directly. The heat transfer model of heating system for $1.2 \mathrm{MW}$ wind turbine blade is built, and influencing analysis of size parameters on heating effect to mold heating system, such as thickness of heat conduction layer, thickness of insulating layer, inner diameter of parallel pipeline, wall thickness of pipe and distance between pipes are accomplished in this paper. The conclusion provides reference for determining variables and their span of system optimum design.
\end{abstract}

Keywords: Heat transfer model, influencing analysis, mold heating effect, size parameter, wind turbine blade.

\section{INTRODUCTION}

As the critical component of capturing wind energy, good quality and superior performance of wind turbine blade is the crucial factors to guarantee its regular and stable running of wind turbine system [1,2]. With the development of wind turbine blade towards to large power, light weight, high performance ratio and low cost, in order to improve its performance, quality and fatigue life, large-scale wind turbine blades is basically made of reinforced material, and manufactured through composite placement and thermosetting base-resin permeability [3].

Curing is one of the important processes in its production of large-scale composite wind turbine blade. Mold's heating system of circulating water is that metal tubes and pipes is embedded in composite moulding surface, and resin is cured through heating transfer of circulating hot water. This heating mode is superior to others, such as cheap thermal medium of high heat capacity, low cost of manufacture and operation, bilateral control mold's temperature and long working life of the mold, and even high quality and production efficiency in curing blade $[4,5]$. The purpose of this paper is to optimize design of this mold heating system through analyzing influence and changing trend different size parameters to mold heating effect.

\section{ESTABLISHING HEAT TRANSFER MODEL OF CIRCULATING WATER HEATING SYSTEM}

3D mold model of circulating water heating system for blade root is shown in Fig. (1). Cross section of 3D model can be used as heat transfer analysis model. Fig. (2). shows the cross section of mold heat system.

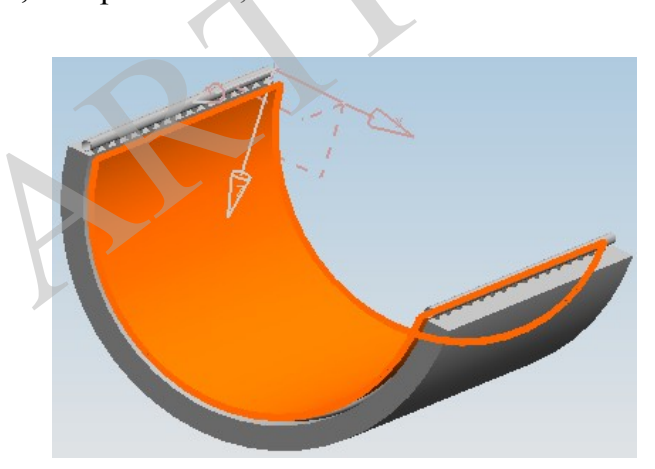

Fig. (1). Model of circulating water heating for blade root.

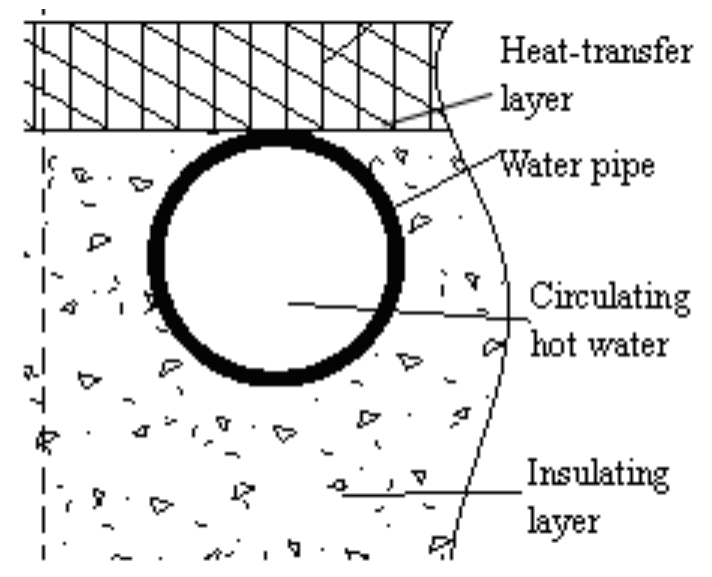

Fig. (2). Cross-section of mold heating system.

Not regarding the heat transfer influence of mold boundary, according to the symmetrical characteristic of heat transfer model, the symmetry unit in Fig. (2) was used as the numerical analysis model of heat transfer, as showed in Fig. (3). Model size is controlled by five parameters of system element. That is thickness of heat conduction layer d, thickness of insulating layer $b$, inner diameter $\Phi$ of parallel pipeline, wall thickness of pipeline $\delta$ and distance between pipes L [6]. 


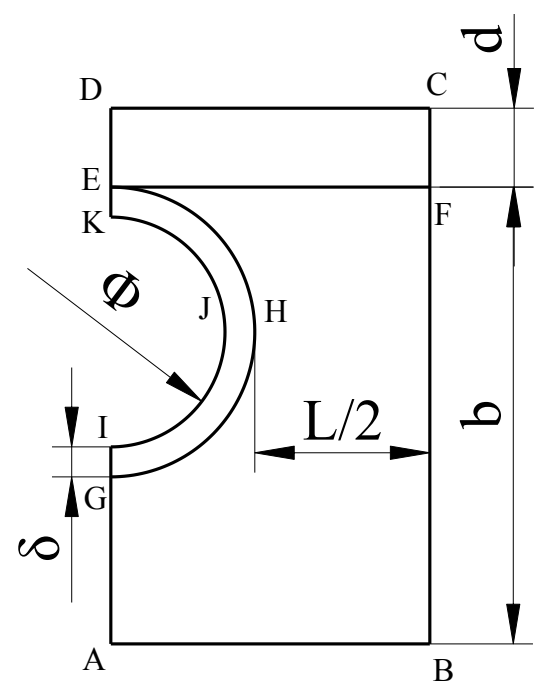

Fig. (3). Numerical analysis model of heat transfer.

\section{INFLUENCE ANALYSIS OF SIZE PARAMETER ON HEATING EFFECT}

Index parameters evaluated heating effect of mould is: 1 . average temperature of moulding surface in steady-state $T_{d}$. Its value expresses heating temperature range of mould. The bigger of $T_{d}$ is, the bigger of heating temperature range is. 2. maximum temperature difference of moulding surface in steady-state $\Delta \mathrm{T}$. The smaller of $\Delta \mathrm{T}$ is, the more even of temperature field is. 3. average temperature of exterior surface of insulating layer $T_{b}$, The smaller of $T_{b}$ is, the better of heat insulating effect is. 4. time from starting heat to steady state $t_{w}$, the smaller $t_{w}$ is, the shorter of curing time and the higher of production efficiency. 5. average heating-up speed rate of mold surface before steady state v. The bigger of $\mathrm{v}$ is, the better of its heat conductivity, the more sensitive of temperature adjusting, and the better of heating effect $[7,8]$.

Hypothesis that heat system reaches steady state when temperature change rate of point $\mathrm{C}$ equals to $0.001^{\circ} \mathrm{C} / \mathrm{s}$.

\subsection{Influence Analysis of Thickness of Heat Conduction Layer on Heating Effect}

Model parameter is as follows: water temperature $\mathrm{T}_{\text {water }}=70{ }^{\circ} \mathrm{C}$, air temperature $\mathrm{T}_{\text {air }}=20^{\circ} \mathrm{C}, \Phi=10 \mathrm{~mm}, \delta=1$ $\mathrm{mm}, \mathrm{L}=22 \mathrm{~mm}, \mathrm{~b}=18 \mathrm{~mm}$. Red copper was used as tubes and pipes, glass microsphere and resin (mass ratio is 1:1) were used as insulating layer, and stream current state is laminar current. Analysis results are shown as in Fig. (4) when d is $3 \mathrm{~mm}, 5 \mathrm{~mm}, 7 \mathrm{~mm}, 9 \mathrm{~mm}$ and $11 \mathrm{~mm}$ respectively.

Through analysis we know that with the decreasing of thickness of heat conduction layer $d, T_{d}$ and $v$ increase, $T_{b}$ and $t_{w}$ decrease. Heating effect improves. But with the decreasing of $b, \Delta \mathrm{T}$ increases obviously. Heating effect of mould decreases. Because $\Delta \mathrm{T}$ is one of the most important index in evaluating heating effect of mold, reasonable value of $\mathrm{d}$ should be selected in design.
T-d

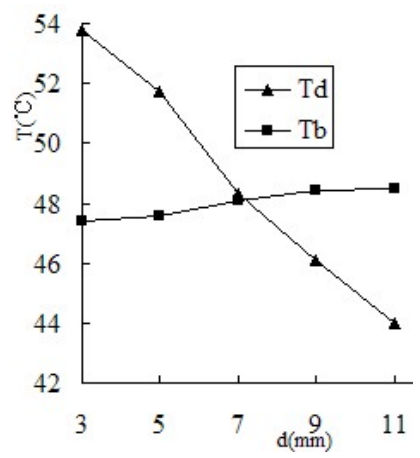

$\Delta \mathrm{T}-\mathrm{d}$
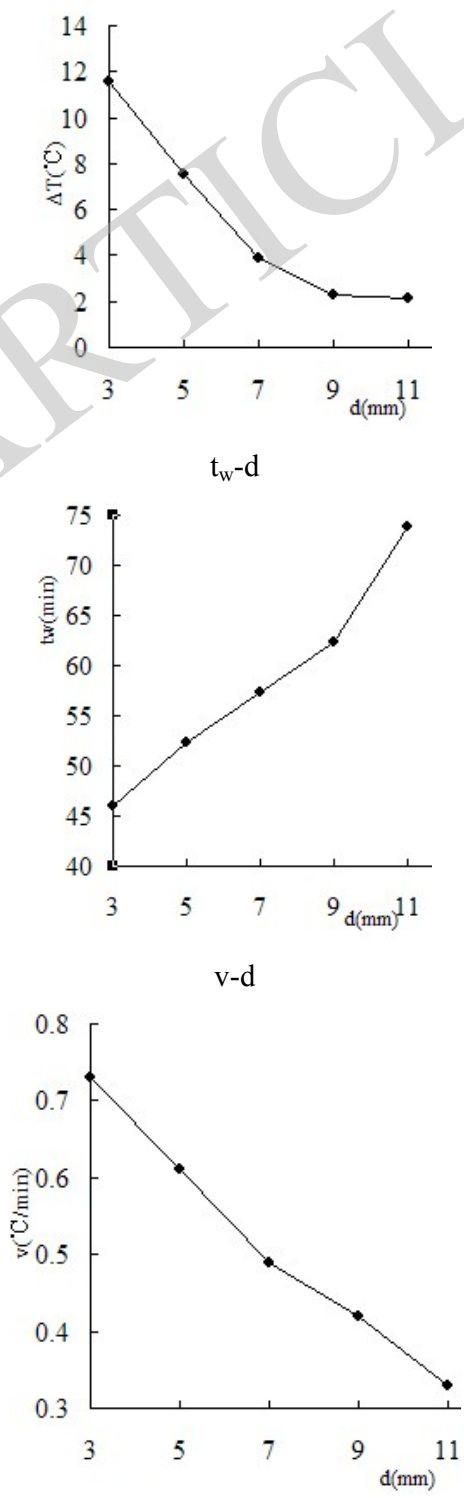

Fig. (4). Influencing evaluation index diagram of thermal layer thickness. 


\subsection{Influence Analysis of Insulating Layer's Thickness on Heating Effect}

Model parameter is as follows: water temperature $\mathrm{T}_{\text {water }}=75^{\circ} \mathrm{C}$, air temperature $\mathrm{T}_{\text {air }}=25^{\circ} \mathrm{C}, \Phi=10 \mathrm{~mm}, \delta=1$ $\mathrm{mm}, \mathrm{L}=20 \mathrm{~mm}, \mathrm{~d}=6 \mathrm{~mm}$. Analysis results are shown as in Fig. (5) when b is $15 \mathrm{~mm}, 17 \mathrm{~mm}, 19 \mathrm{~mm}, 21 \mathrm{~mm}$ and 23 $\mathrm{mm}$ respectively.

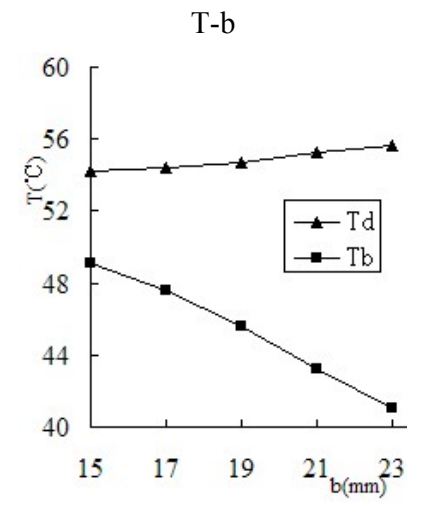

$\Delta \mathrm{T}-\mathrm{b}$
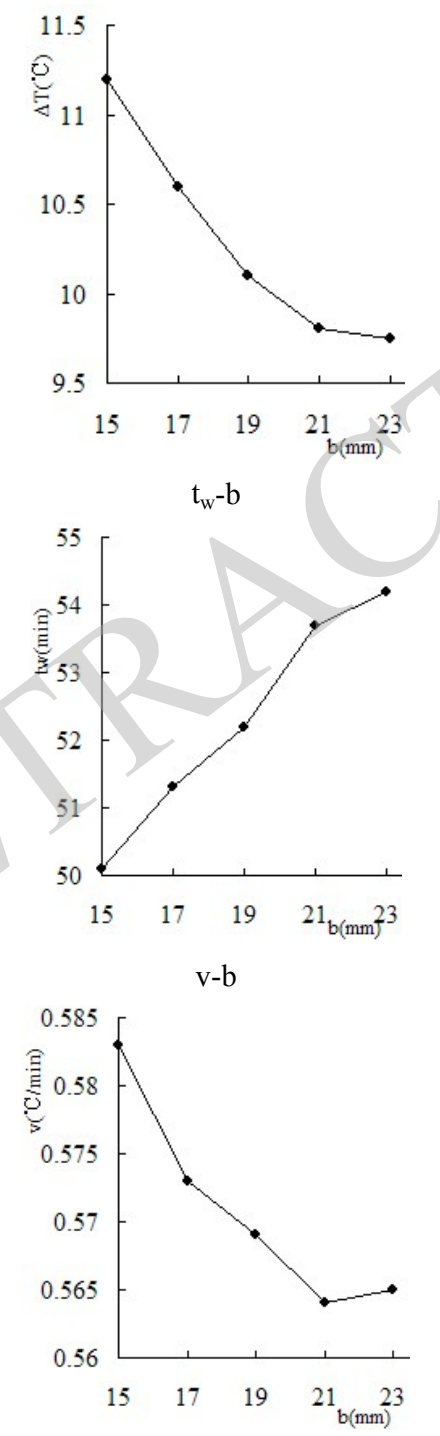

Fig. (5). Influencing evaluation index chart of insulating layer thickness.
Through analysis we know that the bigger $\mathrm{b}$ is, the bigger thermal resistance from heat resource to mould back is, and the smaller heat loss. $t_{\mathrm{w}}$ increases and $\Delta \mathrm{T}$ decreases with it. $T_{d}$ is almost invariant because heat resistance from heat resource to mold surface isn't influenced by $b$ value. According to Fig. (5), the variation of b has little influence to heating effect of mould.

\subsection{Influence Analysis of Inner Diameter of Parallel Pipeline on Heating Effect}

Model parameter is as follows: water temperature $\mathrm{T}_{\text {water }}=80^{\circ} \mathrm{C}$, air temperature $\mathrm{T}_{\text {air }}=15^{\circ} \mathrm{C}, \delta=1 \mathrm{~mm}, \mathrm{~L}=24$ $\mathrm{mm}, \mathrm{d}=5 \mathrm{~mm}, \mathrm{~b}=22 \mathrm{~mm}$. Analysis results are shown as in Fig. (6) when $\Phi$ is $6 \mathrm{~mm}, 12 \mathrm{~mm}, 18 \mathrm{~mm}, 24 \mathrm{~mm}$ and $30 \mathrm{~mm}$ respectively.

Through analysis we know that the bigger $\Phi$ is, the bigger thermal resistance from heat resource to mould surface is. As the enhanced strength of heat resource offset its influence, $T_{d}$ keeps essentially constant. Thermal conduction distance from heat reservoir to out-surface of insulating layer decrease with the increase of $\Phi$. Thermal resistance decreases and $T_{b}$ increases follow it. When $\Phi$ increases, longitudinal thermal resistance of mould surface is not changed, and horizontal thermal resistance increases. Thermal resistance difference of mould surface decreases [9], and $\Delta T$ increases. Diffusion distance increases with the increasing of $\Phi$, but thermal diffusivity isn't changed, so $t_{w}$ increases. $\mathrm{V}$ is in inverse proportion to $t_{w}$ because of the stabilization of $T_{d}$. So decreasing $\Phi$ can improves the heating effect of mould.

\subsection{Influence Analysis of Wall Thickness of Pipeline on Heating Effect}

Model parameter is as follows: water temperature $\mathrm{T}_{\text {water }}=75^{\circ} \mathrm{C}$, air temperature $\mathrm{T}_{\mathrm{air}}=20^{\circ} \mathrm{C}, \mathrm{L}=24 \mathrm{~mm}, \mathrm{~d}=5$ $\mathrm{mm}, \mathrm{b}=22 \mathrm{~mm}$. Analysis results are shown as in Fig. (7). when $\delta$ is $0.5 \mathrm{~mm}, 1.0 \mathrm{~mm}, 1.5 \mathrm{~mm}, 2.0 \mathrm{~mm}$ and $2.5 \mathrm{~mm}$ respectively.

Through analysis we know that thermal resistance of pipe wall increases with the increasing of $\delta$, and total thermal resistance of mould surface keeps unchanged basically, so $T_{d}$ keeps constant. The increasing of $\delta$ leads to the decrease of average thickness of insulating layer, and total thermal resistance from heat resource to out-surface of insulating layer decreases, so $\mathrm{T}_{\mathrm{b}}$ increases. The increasing of $\delta$ leads to the increase of horizontal diffusion distance of mould surface, so the lowest temperature of mould surface decreases. Because stable heat reservoir can't change the highest temperature of mould surface, $\Delta \mathrm{T}$ increases. $\mathrm{t}_{\mathrm{w}}$ and $\mathrm{v}$ is unchanged basically, so the decreasing of $\delta$ can improves the heating effect of mould.

\subsection{Influence Analysis of Distance Between Pipes on Heating Effect}

Model parameter is as follows: water temperature $\mathrm{T}_{\text {water }}=80^{\circ} \mathrm{C}$, air temperature $\mathrm{T}_{\text {air }}=15^{\circ} \mathrm{C}, \Phi=24 \mathrm{~mm}, \mathrm{~d}=5$ $\mathrm{mm}, \mathrm{b}=22 \mathrm{~mm}$. Analysis results are shown as in Fig. (8) when $\mathrm{L}$ is $6 \mathrm{~mm}, 12 \mathrm{~mm}, 18 \mathrm{~mm}, 24 \mathrm{~mm}$ and $30 \mathrm{~mm}$ respectively. 
$\mathrm{T}-\Phi$
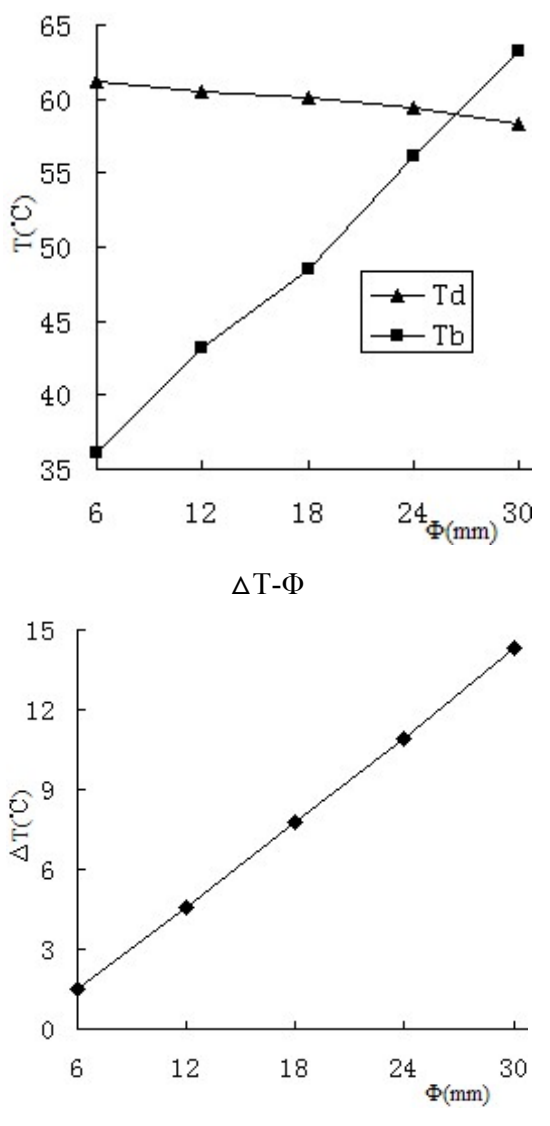

$\mathrm{t}_{\mathrm{w}}-\Phi$
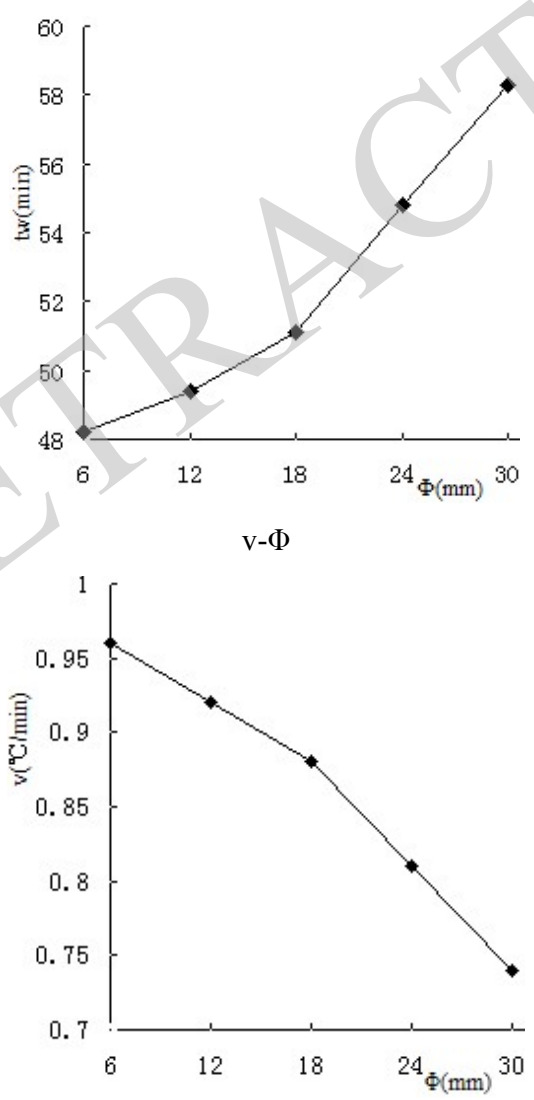

Fig. (6). Influencing evaluation index chart of pipeline inner diameter.
$\mathrm{T}-\delta$

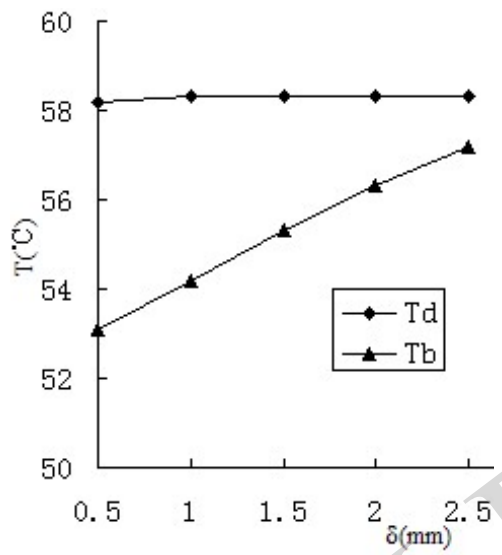

$\Delta \mathrm{T}-\delta$

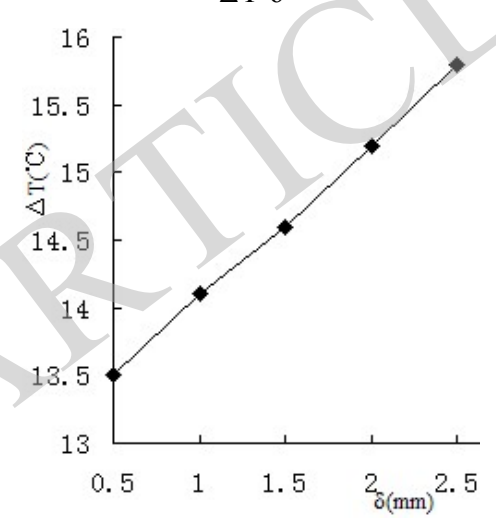

$t_{w}-\delta$
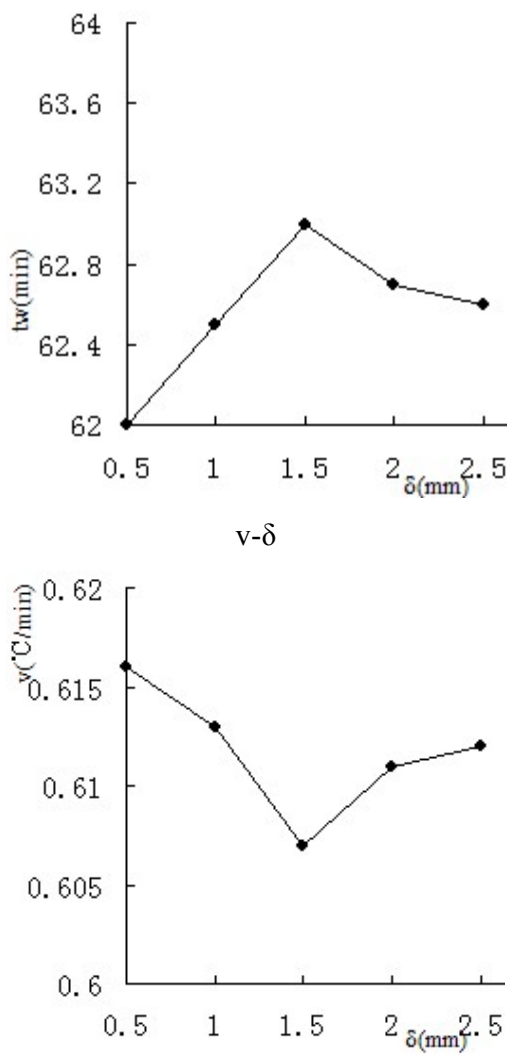

Fig. (7). Influencing evaluation index chart of pipeline wall thickness. 
$\mathrm{T}-\mathrm{L}$

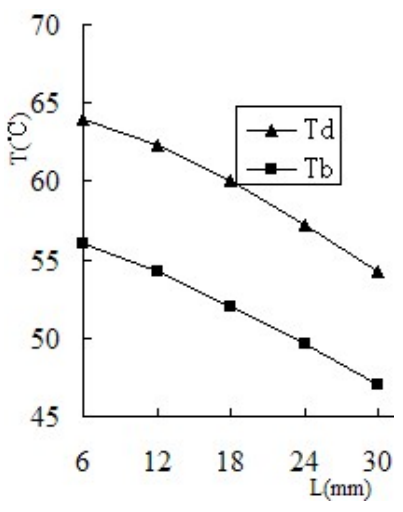

$\Delta \mathrm{T}-\mathrm{L}$

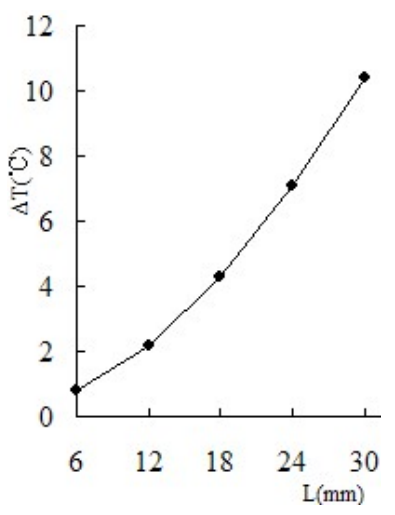

$t_{w}-L$

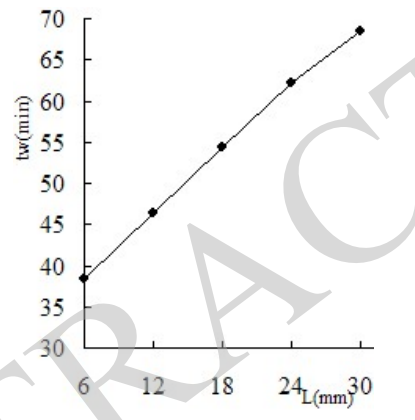

$\mathrm{V}-\mathrm{L}$

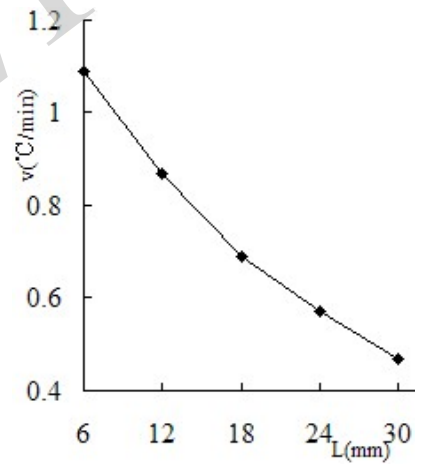

Fig. (8). Influencing evaluation index chart of pipe spacing.
Through analysis we know that the increasing of L leads to the increasing of horizontal diffusion distance, and thermal resistance of mould surface and out-surface of insulating layer increases both, so $T_{d}$ and $T_{b}$ decrease both. The increasing of $\mathrm{L}$ leads to the increasing of horizontal thermal resistance of mould surface, but longitudinal thermal resistance keeps unchanged, so $\Delta \mathrm{T}$ increases. Horizontal diffusion distance increases with the increasing of $\mathrm{L}$, but diffusion coefficient keeps unchanged, so $t_{w}$ increases. When $t_{w}$ increases, $T_{d}$ decreases and $t_{w}$ increases, so $v$ decreases. Therefore the decreasing of $\mathrm{L}$ can improve the heating effect of mould.

\section{CONCLUSION}

Through the influencing analysis of size parameters on heating effect for mold heating system, we know that the decreasing of $\delta, \Phi$ and $\mathrm{L}$ can improve the heating effect of mould. The changing of $b$ has little influence to heating effect of mould. If $\mathrm{d}$ decreases, tw decreases also, $\mathrm{v}$ and $\mathrm{Td}$ increases, besides, $\Delta \mathrm{T}$ increases also. So the span of $\mathrm{d}$ should be reasonable range. The conclusion provides reference for determining variables and their span of system optimum design.

\section{CONFLICT OF INTEREST}

The authors confirm that this article content has no conflict of interest.

\section{ACKNOWLEDGEMENTS}

This research has received financial support by the Inner Mongolia Natural Science Foundation of China (2014MS0514).

\section{REFERENCES}

[1] B.S. Pan, S.J. Xie, and L.H. Liang, "Research of composite ply strength properties for the root of wind turbine blade", Acta Energiae Solaris Sinica, vol. 33, pp. 769-775, May 2012.

[2] P. Srensen, "Wind models for simulation of power fluctuations from wind farms", J. Wind Eng. Ind. Aerodyn., vol. 15, pp. 13981399, May 2012.

[3] S. J. Chen, Z. S. Shen, and H.S. Xu, "Composites and wind turbine blades", Renewable Energy Resource, vol. 26, pp. 90-92, April 2008.

[4] X.B. Feng, and W. Wang, "Calefaction research and technology innovation of 1.5 megawatt wind turbine bland mould", Fiber Reinforced Plastics/Composites, pp. 66-68, vol. 88, May 2010.

[5] L.M. Stern, "Advances in electron-beam processing of fiberreinforced plastics: an emerging curing technology", SAMPE J., vol. 36, pp. 33-38, March-April 2000.

[6] C.H. Dai, "Design of Large-Scale Composite Mould with Inside Circulating Water Heating System," Master thesis, National University of Defense Technology, Changsha, China, November, 2007.

[7] G.L. Wang, G.Q. Zhao, and H.P. Li, "Analysis of thermal cycling efficiency and optimal design of heating/cooling systems for rapid heat cycle injection molding process", Mater. Des., vol. 31, pp. 3426-3441, August 2010. 
[8] G.L. Wang, G.Q. Zhao and H.P. Li, "Research on optimization design of the heating-cooling channels for rapid heat cycle molding based on response surface methodology and constrained particle swarm optimization", Expert Syst. Appl., vol. 38, pp. 6705-6719, June 2011.
[9] X.B. Ma, "Research on Thermal Characteristic about Headstock of SYSK-209 Pipe Threading Lathe", Master thesis, Jilin University, Changchun, China, May, 2006.

Received: January 8, 2015

(C) Sun et al.; Licensee Bentham Open.

This is an open access article licensed under the terms of the Creative Commons Attribution Non-Commercial License (http://creativecommons.org/licenses/by-nc/3.0/) which permits unrestricted, non-commercial use, distribution and reproduction in any medium, provided the work is properly cited. 